Recepción: 20 / 04 / 2017

Aceptación: 20 / 05 / 2017

Publicación: 15 / 06 / 2017

Seguridad Industrial

Artículo de Investigación

\title{
El ruido como factor de riesgo físico y su incidencia en la calidad de vida de los trabajadores en la Productividad organizacional
}

\author{
The noise like the physical risk factor and its incidence \\ in the quality of life of the workers in the Organizational Productivity
}
El ruído como risco físico e seu impacto sobre a qualidade de vida dos trabalhadores da produtividade organizacional

\author{
William F. Salcedo-Medrano I \\ william.salcedo@tonicorp.com \\ Héctor D. Martínez-Villacrés II \\ hector.martinezvi@ug.edu.ec \\ Edin A. Garcés-Coca III \\ edin.garcesc@ug.edu.ec
}

Correspondencia: william.salcedo@tonicorp.com

I. Ingeniero Químico; Especialista en Producción más Limpia, Magister en Seguridad Higiene Industrial y Salud Ocupacional, Diploma Superior en Seguridad Higiene y Salud Ocupacional, Universidad Estatal de Guayaquil, Guayaquil, Ecuador.

II. Ingeniero en Sistemas; Magister en Docencia Matemática, Universidad Estatal de Guayaquil, Guayaquil, Ecuador.

III. Ingeniero Aeronáutico; Especialidad "Navegación Aérea y Control de Tránsito Aéreo" Magister en Ciencias de Ingeniería, Universidad Estatal de Guayaquil, Guayaquil, Ecuador. 


\section{Resumen}

El propósito de este trabajo es formular un modelo de gestión para las empresas que les permita identificar de como el Ruido tiene incidencia en la calidad de vida de los trabajadores así como en su productividad. Durante la realización de este estudio en las diferentes áreas productivas se identificaron fuentes de ruido que podrían generar daño auditivo o generar efectos extra-auditivos que pueden incidir en los aspectos fisiológicos, psicológicos y sociales de los trabajadores expuestos. Para el análisis de los datos se elaboró un base de datos que permite calcular de manera dinámica el tiempo permitido, la dosis parcial, la dosis total y los NPS (e); con el cálculo de la dosis se pueden definir criterios de actuación para trabajadores estándar y trabajadores vulnerables. El resultado de una evaluación de ruido nos ayudó hacer un inventario de acciones, con el fin de diseñar, mantener o mejorar los controles de riesgos, especialmente para aquellos valores cuya dosis se encuentran por encima de 1 .

Palabras claves: Ruido, Sonómetro, Gestión, Sistema, Vigilancia, Dosis, Seguridad, Higiene, Industrial, Salud, Ocupacional. 


\begin{abstract}
The purpose of this paper is to formulate a management model for companies that allows them to identify how noise has an impact on the quality of life of workers as well as their productivity. During the realization of this study in the different productive areas, noise sources were identified that could generate auditory damage or generate extra-auditory effects that can affect the physiological, psychological and social aspects of exposed workers. For the analysis of the data, a database was created that allows to calculate in a dynamic way the allowed time, the partial dose, the total dose and the NPS (e); With the calculation of the dose can be defined criteria of action for standard workers and vulnerable workers. The result of a noise assessment helped us to make an inventory of actions, in order to design, maintain or improve risk controls, especially for those values whose dosage is above 1 .
\end{abstract}

Key words: Noise, Sound level meter, Management, System, Surveillance, Dose, Safety, Hygiene, Industrial, Health, Occupational. 


\section{Resumo}

Objetivo deste trabalho é desenvolver um modelo de gestão para as empresas que lhes permitem identificar como o ruído tem um impacto sobre a qualidade de vida dos trabalhadores e sua produtividade. Durante a realização deste estudo nos diferentes fontes áreas de produção de ruído que podem causar danos auditivos ou geram efeitos extra-auditivos que podem afetar foram identificados os aspectos fisiológicos, psicológicos e sociais dos trabalhadores expostos. Para analisar os dados, um banco de dados calcula dinamicamente o tempo permitido, a dose parcial, a dose total e o NPS (e) foi preparado; com cálculo da dose pode ser definido critérios de desempenho para trabalhadores padrão e trabalhadores vulneráveis. O resultado de uma avaliação do ruído nos ajudou a fazer um inventário de ações, a fim de projetar, manter ou melhorar os controles de risco, especialmente para aqueles cujos valores de dose são superiores a 1.

Palavras-chave: Ruído, Medidor de Nível Sonoro, gestão, acompanhamento do sistema, dose, saúde, segurança, industriais, saúde, ocupacional. 


\section{Introducción.}

El formular un modelo de gestión del factor de Riesgo Físico: Ruido y establecer la incidencia sobre calidad de vida de los trabajadores así como en la productividad de la organización nos permitirá establecer una metodología sistémica que incluye la participación, inclusión, análisis técnico, competencias del recurso humano y el desarrollo de un proceso de mejoramiento continuo. (Ballesteros \& Daponte, 2011) (Cabaleiro Postela, 2010) (Díaz Saso, 2009)

Para cumplir con los objetivos planteados el presente trabajo parte de un estudio bibliográfico científico que permite alcanzar y desarrollar el tema con base a hechos y datos validados, es muy importante analizar y conocer la legislación vigente relacionadas al factor de Riesgo Físico: Ruido así como conocer metodología de cálculo que va a generar información que permitirá gestionar los resultados del estudio. (Chavez, 2010)

Los conocimientos bibliográficos y de legislación así como la experiencia del autor permitió desarrollar una metodología apropiada para planificar, determinar recursos, desarrollar la parte experimental, recolectar datos y aplicar herramientas estadísticas que permitan analizar los resultados obtenidos así como definir las acciones a tomar de acuerdo a su significancia. La implementación de un sistema de gestión estaría incompleta si no existe un proceso de auditorías y mejoramiento continuo. (Näf Cortés, 2013) (Ramos Fernández, 2013)

Finalmente consideramos que el presente trabajo está centrado en la identificación y prevención de la incidencia factor Riesgo Físico: Ruido en la salud de los trabajadores 


\section{Materiales y métodos.}

El presente trabajo sirvió para establecer un sistema de gestión por medio del cual se establezca la metodología de medición, la recolección, la sistematización y el análisis de datos, después de lo cual se pueda calcular el tiempo máximo permitido, la dosis de exposición y el Nivel de Presión Sonora Equivalente. Los resultados obtenidos permitirán definir las medidas correctivas y preventivas con el fin de controlar los niveles de ruido existentes dentro de cualquier planta productiva y de esta manera minimizar al máximo el riesgo de pérdida de la audición de los trabajadores.

Tipo y diseño de la investigación

Se realizó un estudio descriptivo, en las áreas de proceso y envasado de cinco plantas productoras de alimentos.

\begin{tabular}{|c|c|c|c|c|}
\hline $\begin{array}{l}\text { Codificación } \\
\text { de la } \\
\text { empresa }\end{array}$ & CIIU & $\begin{array}{l}\text { Productos } \\
\text { fabricados }\end{array}$ & $\begin{array}{c}\text { Tipo de } \\
\text { Empresa }\end{array}$ & Ubicación \\
\hline Planta 1 & & $\begin{array}{c}\text { Leche } \\
\text { fermentada }\end{array}$ & Grande & $\begin{array}{c}\text { Guayas } \\
\text { Guayaquil }\end{array}$ \\
\hline Planta 2 & & $\begin{array}{l}\text { Leche de } \\
\text { sabores }\end{array}$ & Grande & $\begin{array}{c}\text { Guayas } \\
\text { Guayaquil }\end{array}$ \\
\hline Planta 3 & & $\begin{array}{c}\text { Jugos larga } \\
\text { vida }\end{array}$ & Grande & $\begin{array}{c}\text { Guayas } \\
\text { Guayaquil }\end{array}$ \\
\hline Planta 4 & & $\begin{array}{c}\text { Jugos larga } \\
\text { vida }\end{array}$ & Mediana & $\begin{array}{c}\text { Guayas } \\
\text { Guayaquil }\end{array}$ \\
\hline Planta 5 & & $\begin{array}{c}\text { Leche } \\
\text { fermentada }\end{array}$ & Medina & $\begin{array}{c}\text { Guayas } \\
\text { Guayaquil }\end{array}$ \\
\hline
\end{tabular}

Tabla $n^{\circ}$ 1.- Características de las empresas. 


\section{Resultados.}

Resultados Nivel de presión sonora NPS (e) por Planta

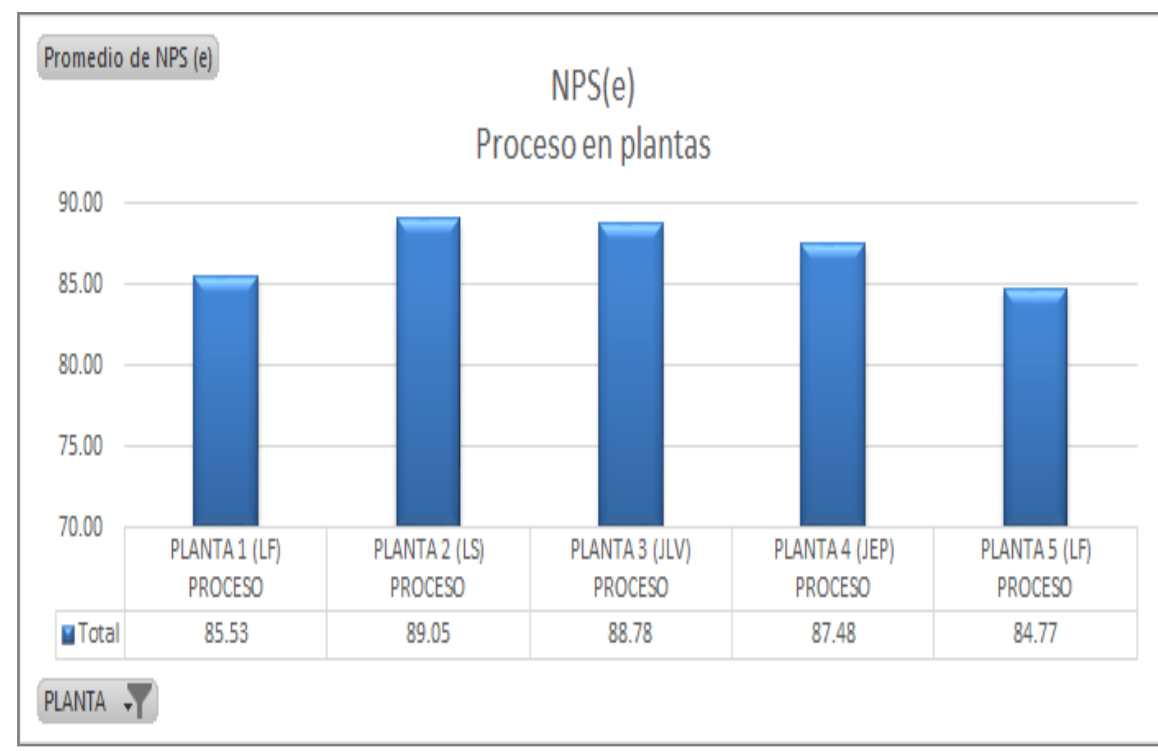

Gráfico n $^{\circ} 1$

Análisis de resultados NPS (e) (proceso)

Fuente: investigación de campo

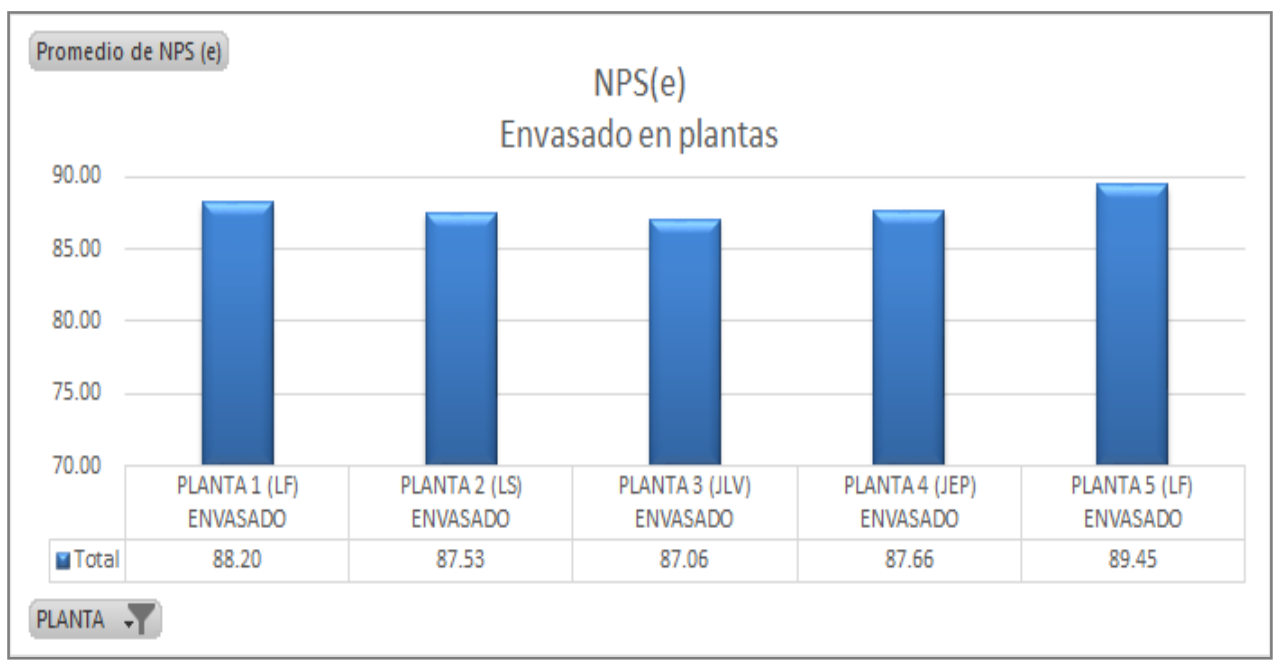

Gráfico n ${ }^{\circ} 2$

Análisis de resultados nps (e) (envasado)

Fuente: investigación de campo 
Resultados Nivel de Dosis por Planta

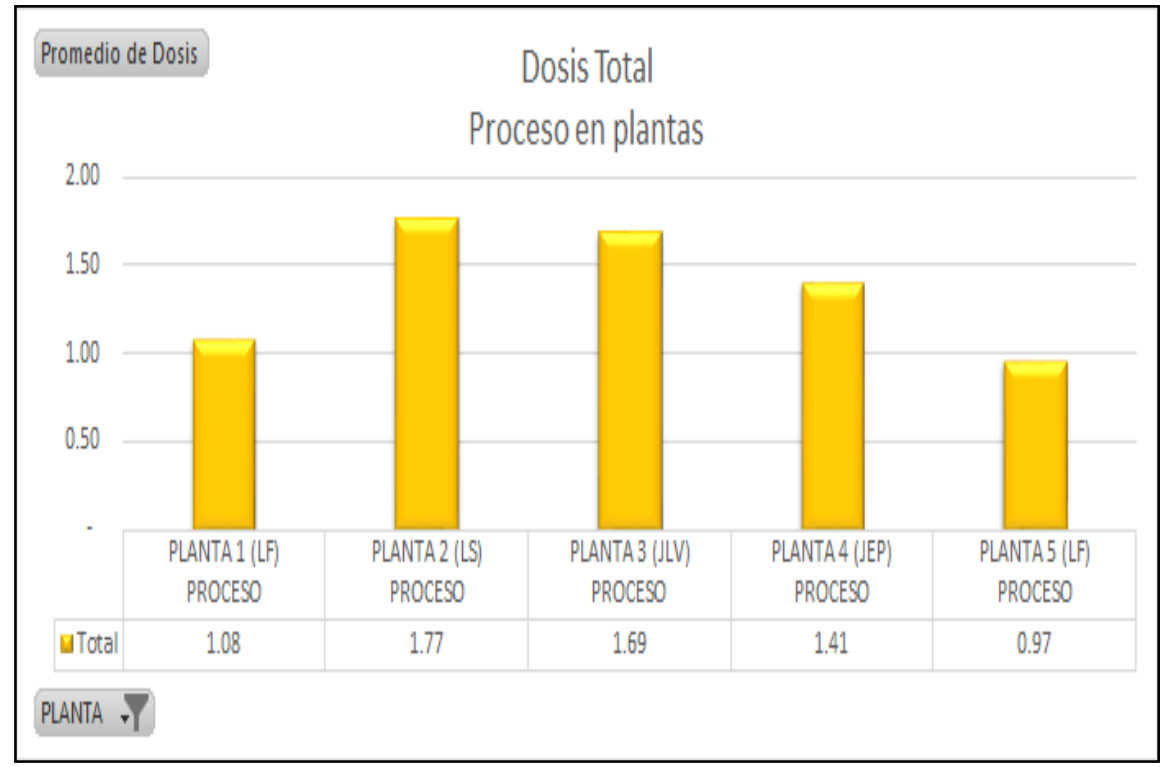

Gráfico $n^{\circ} 3$

Análisis de resultados dosis (proceso)

Fuente: investigación de campo

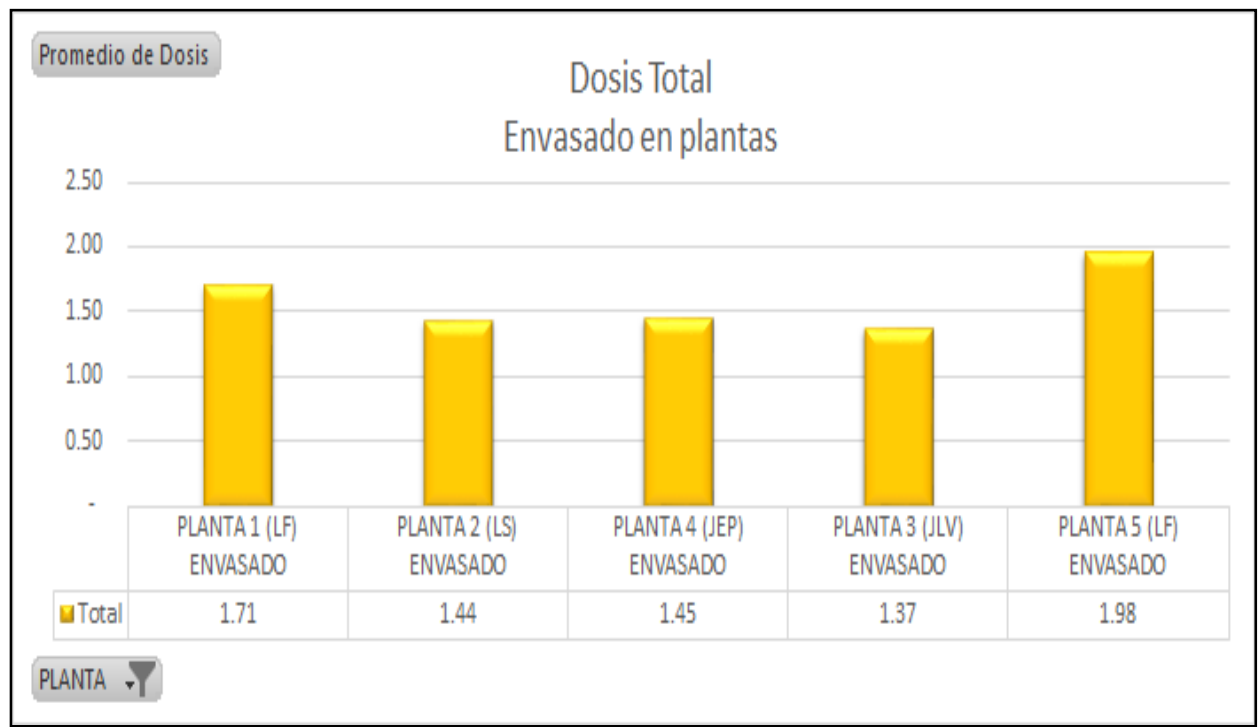

Gráfico n ${ }^{\circ} 4$

Análisis de resultados dosis (envasado)

Fuente: investigación de campo 


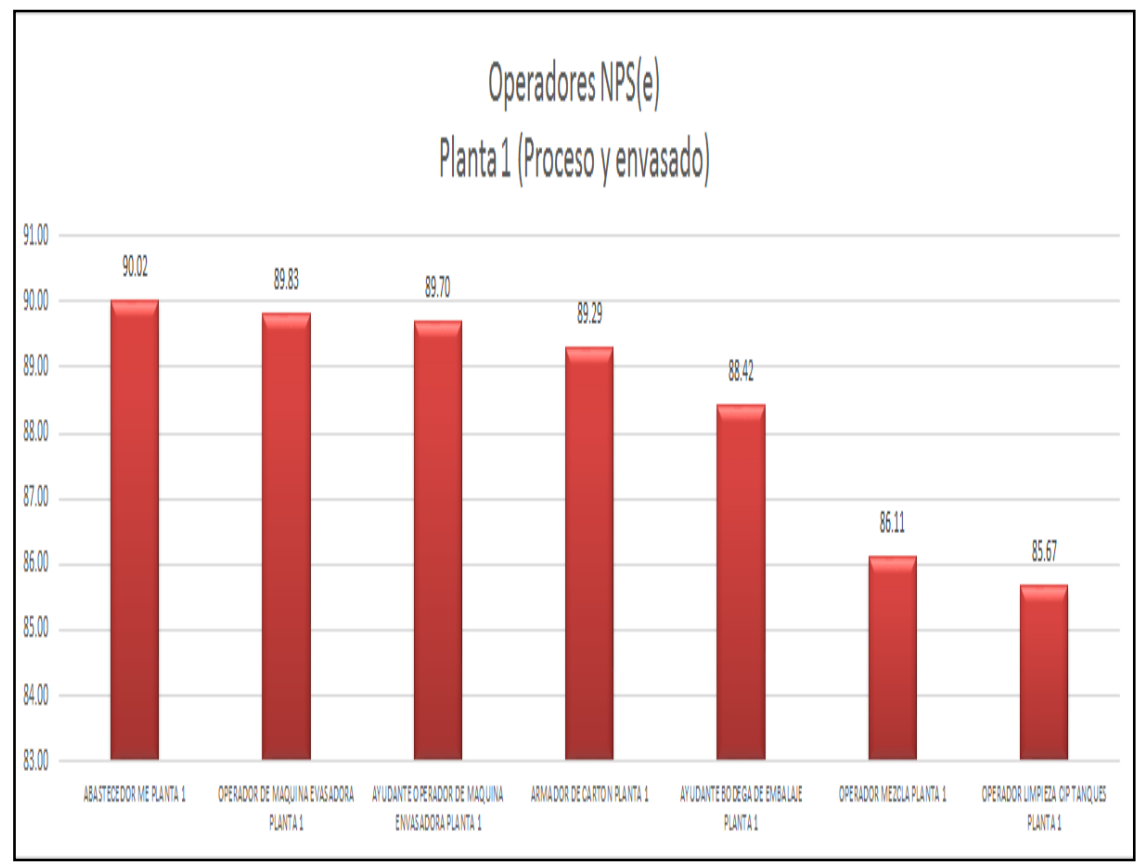

Gráfico $\mathbf{n}^{\circ} 5$

Análisis de resultados NPS (e) operadores planta 1

Fuente: investigación de campo

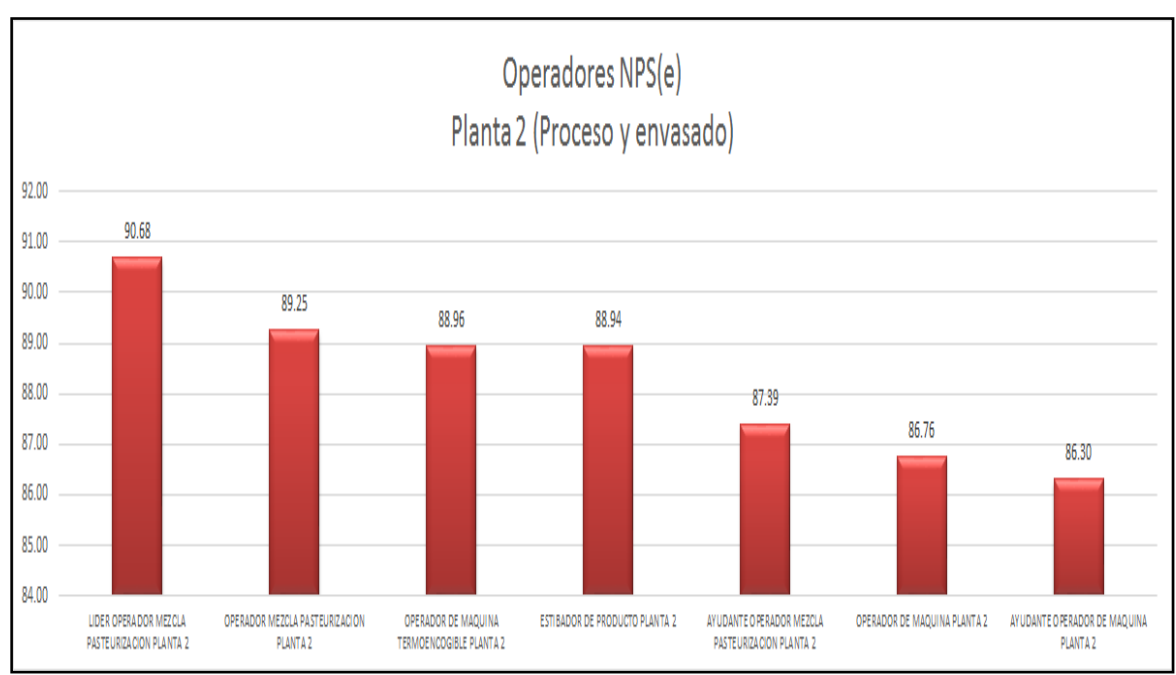

Gráfico $n^{\circ} 6$

Análisis de resultados NPS (e) operadores planta 2

Fuente: investigación de campo 


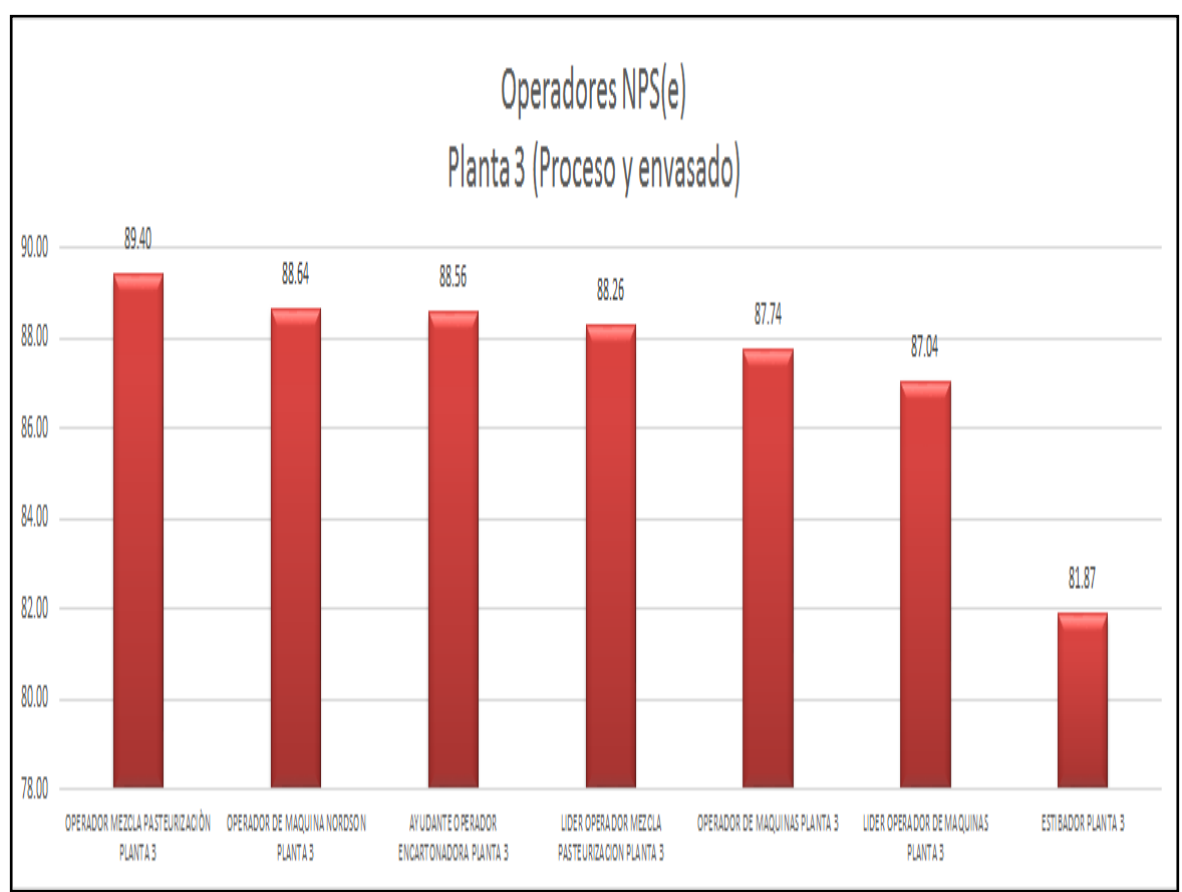

Gráfico n $^{\circ} 7$

Análisis de resultados NPSe) operadores planta 3

Fuente: investigación de campo

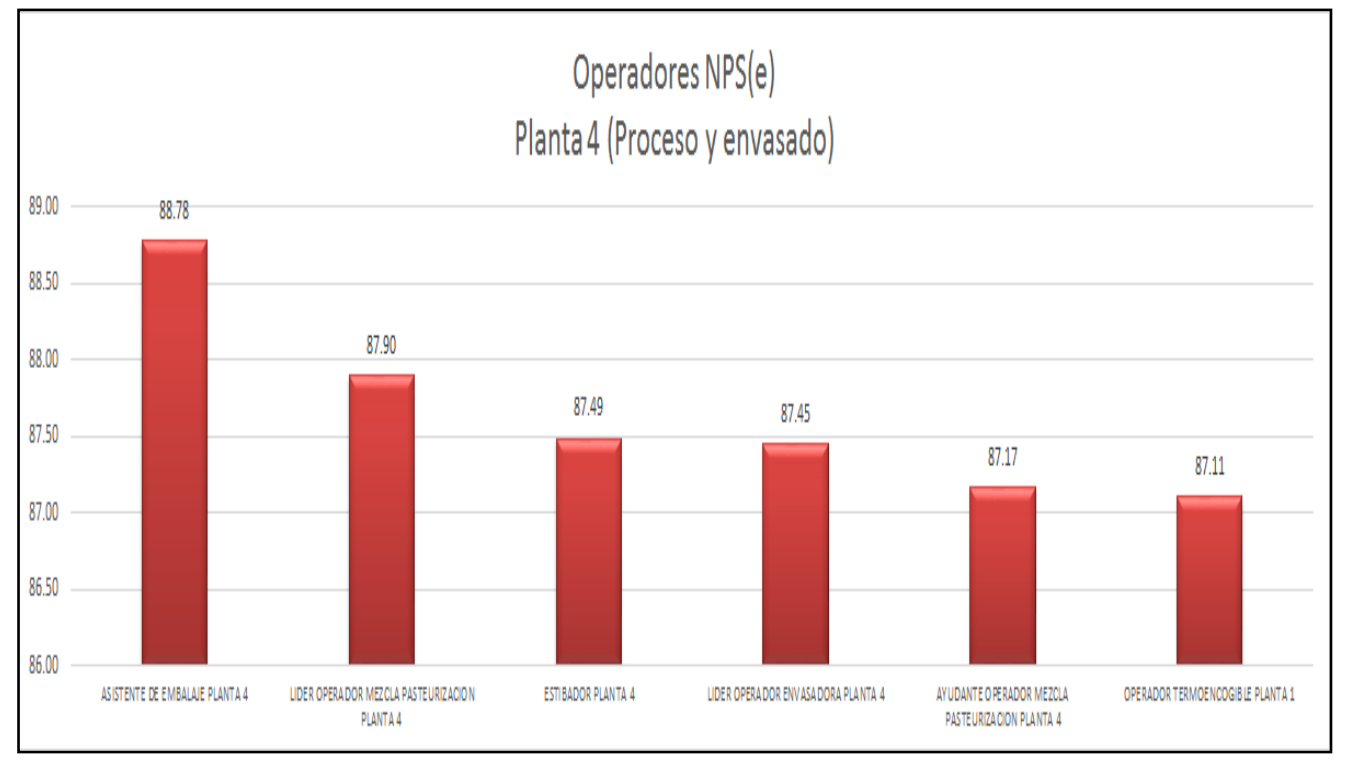

Gráfico n ${ }^{\circ} 8$

Análisis de resultados NPS (e) operadores planta 4

Fuente: investigación de campo 
El ruido como factor de riesgo físico y su incidencia en la calidad de vida de los trabajadores en la Productividad organizacional

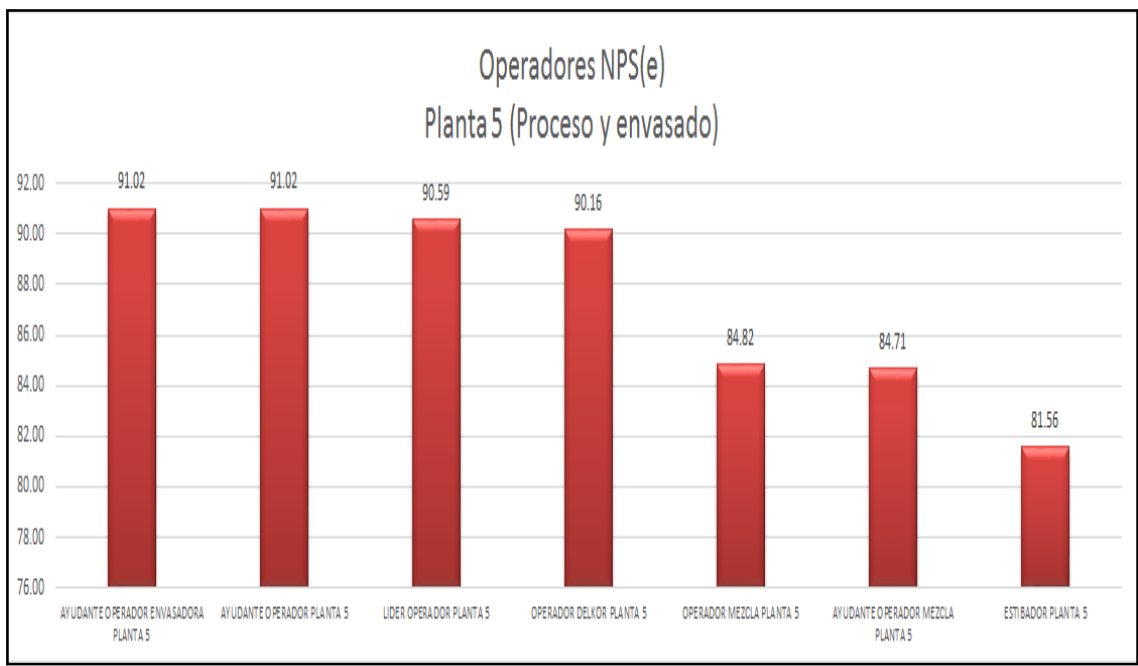

Gráfico $n^{\circ} 9$

Análisis de resultados NPS (e) operadores planta 5

Fuente: investigación de campo

Resultados Nivel de Dosis por Operador

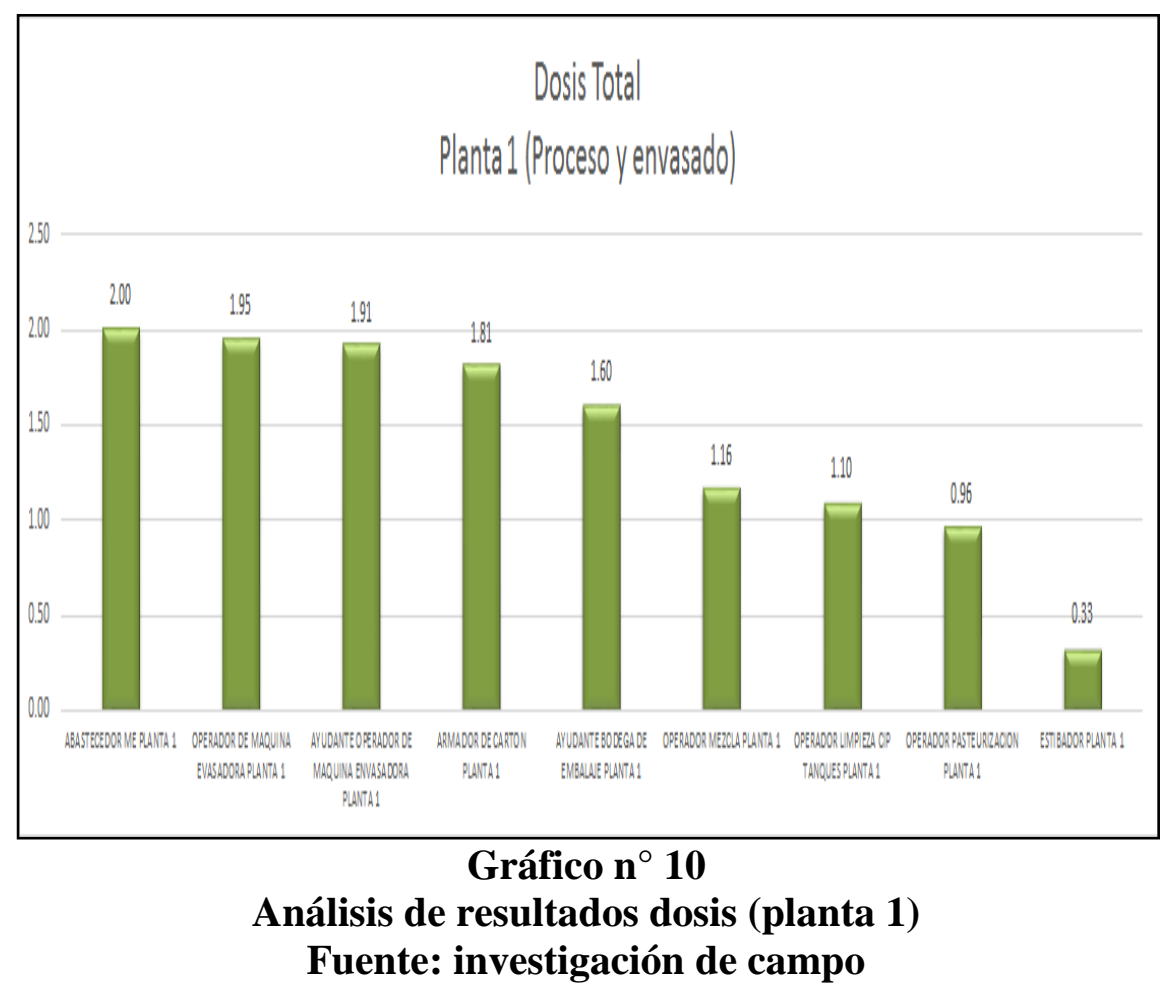

Pol. Con. (Edición núm. 7) Vol. 2, No 6, junio 2017, pp. 755-769, ISSN: 2550 - 682X 


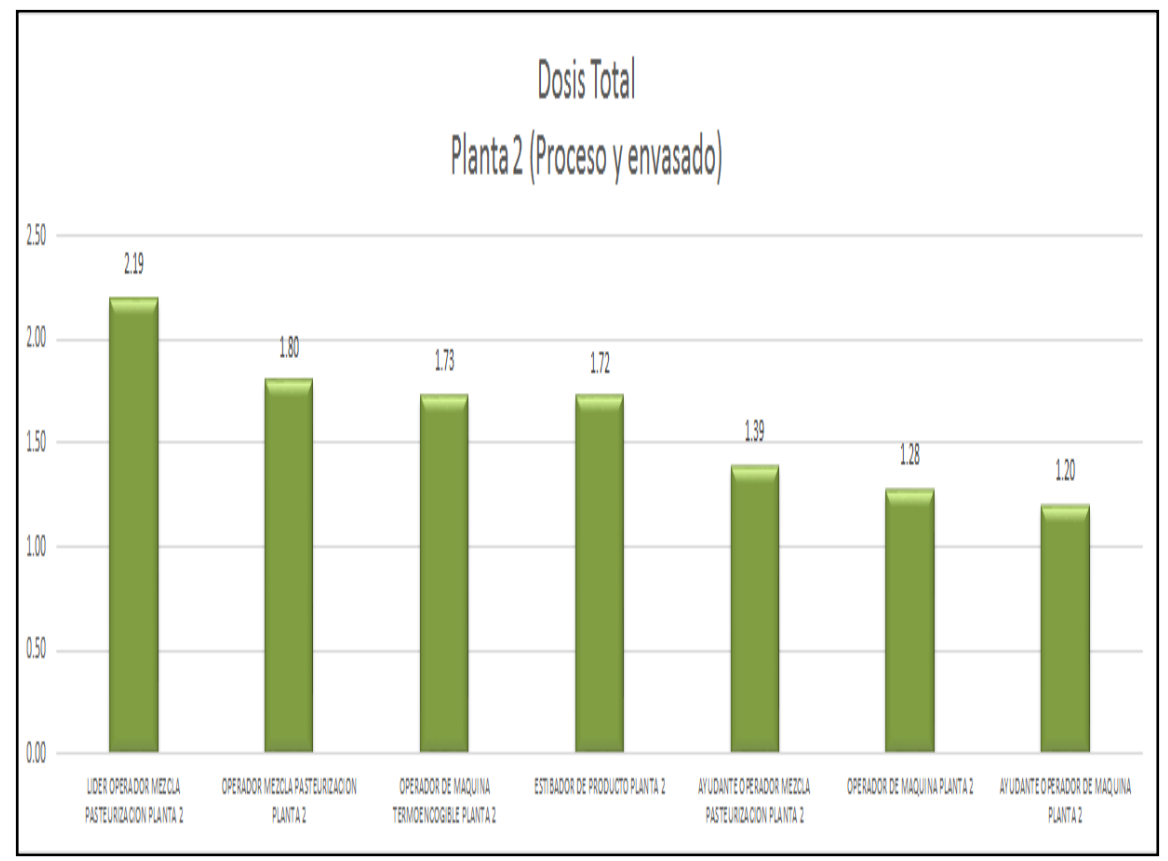

Gráfico n ${ }^{\circ} 11$

Análisis de resultados dosis (planta 2)

Fuente: investigación de campo

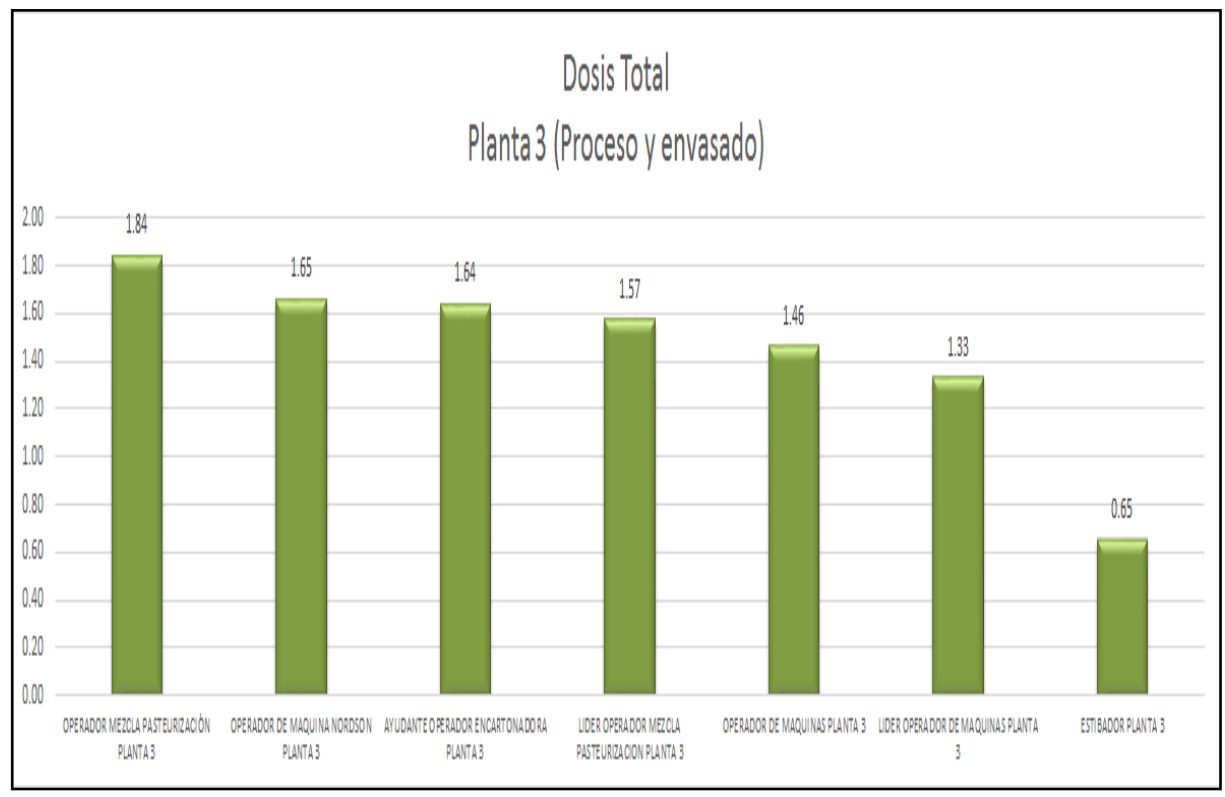

\section{Gráfico n ${ }^{\circ} 12$}

Análisis de resultados dosis (planta 3)

Fuente: investigación de campo 
El ruido como factor de riesgo físico y su incidencia en la calidad de vida de los trabajadores en la Productividad organizacional

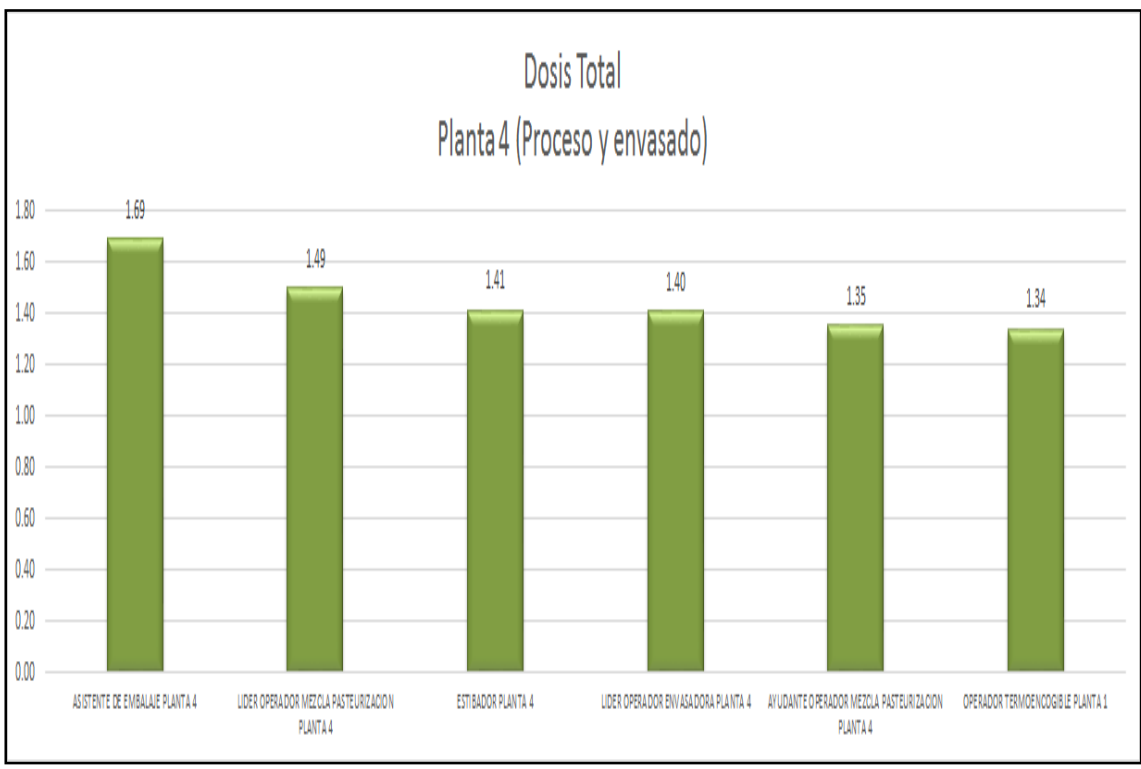

Gráfico n ${ }^{\circ} 13$

Análisis de resultados dosis (planta 4)

Fuente: investigación de campo

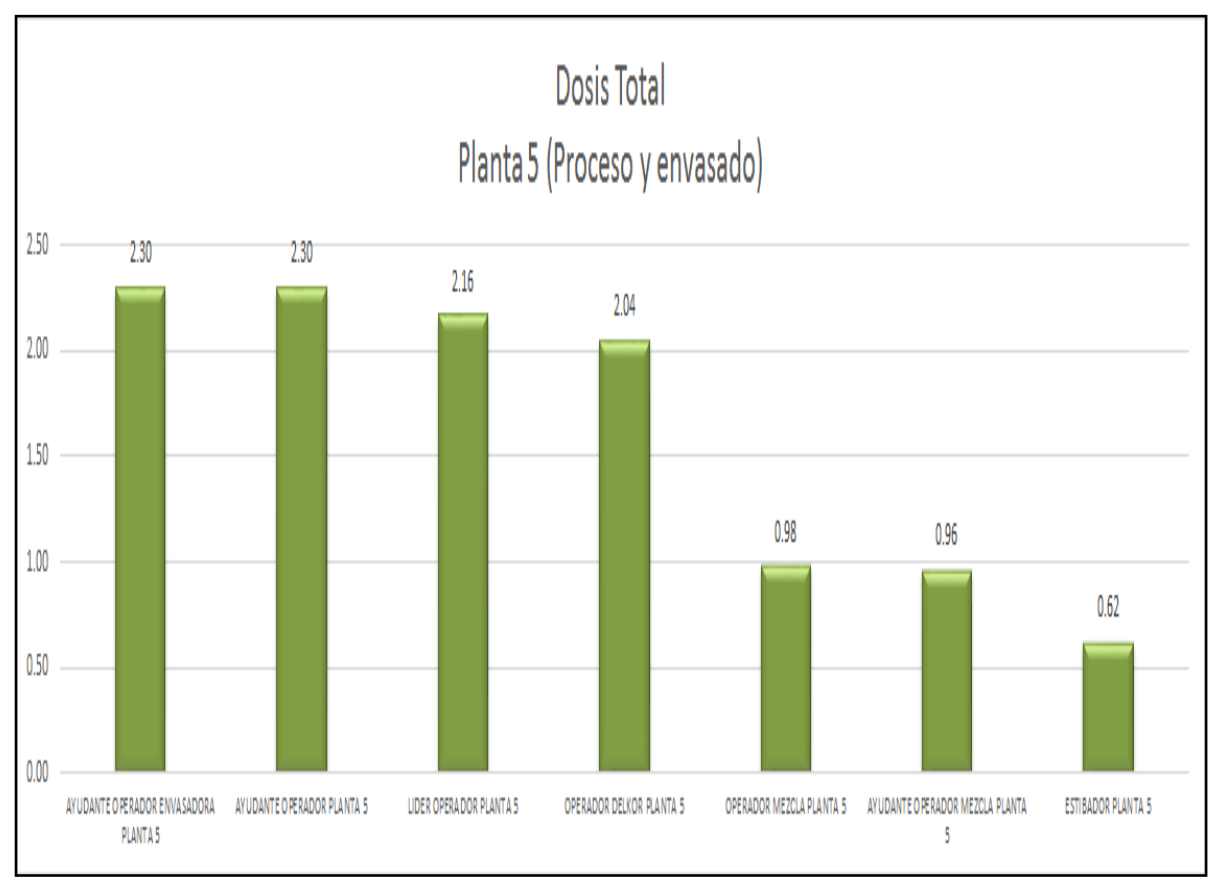

Gráfico n $^{\circ} 14$

Análisis de resultados dosis (planta 5)

Fuente: investigación de campo 


\section{Conclusiones.}

El factor de Riesgo FISICO: RUIDO de acuerdo a los resultados obtenidos en las plantas de proceso de envasado cuya dosis es mayor a 1 influye en temas de productividad por rotación de personal y reubicación de puestos de trabajo.

Las mediciones ambientales por si solas no representan un factor de riesgo; las mediciones ambientales junto con mediciones biológicas determinarán el grado de incidencia del RUIDO sobre la salud del trabajador.

Para trabajadores con dosis menores a 1 cuyos tiempos de exposición son bajos y los niveles de presión sonora altos no garantizan el no desarrollar planes de acción o dotación de EPP's

El $65 \%$ de los trabajadores expuestos a ruido en las diferentes áreas y zonas de las plantas de proceso y envasado se encuentran por encima el nivel de presión sonora establecido por ley.

El $80 \%$ de los trabajadores expuestos a ruido en las diferentes áreas y zonas de las plantas de proceso y envasado se encuentran por encima de la dosis máxima permitida (mayor a 1).

\section{Agradecimientos.}

A la Universidad Estatal de Guayaquil, junto con las autoridades y personal docente de la Facultad de Ingeniería Industrial por la oportunidad para realizar la presente investigación.

\section{Bibliografía.}

Ballesteros, V., \& Daponte, A. (2011). Ruido y Salud. Barcelona: OSMAN.

Cabaleiro Postela, V. (2010). Prevención de riesgos laborales. Madrid: Ideas propias. 
Chavez, J. (2010). Identificación, evaluación y propuesta de control de riesgos labores, aspectos e impactos ambientales en Sección de Fundición de una planta de Grifería Sanitaria. Quito: Universidad Técnica Particular de Loja.

Díaz Saso, P. (2009). Prevención de riesgos laborales. Madrid: Ediciones Nobel.

Näf Cortés, R. (2013). Guía Práctica Para el Análisis y la Gestión del Ruido Industrial. Madrid: FREMAP.

Ramos Fernández, G. (2013). Evaluación de riesgos laborales en un taller mecánico. Almeria: Universidad de Almeria. 